\title{
Caractérisation Du Contenu Pollinique De L'atmosphère De La Commune d'Abomey-Calavi De 2015 À 2017
}

\author{
Tchabi F. Lopez, Msc \\ Tossou G. Monique, PhD \\ Zanou R. Adéline, Msc \\ Akoegninou Akpovi, PhD
}

Laboratoire de Botanique et Ecologie Végétale, Faculté des Sciences et

Techniques, Université d'Abomey-Calavi, Cotonou, Bénin

\section{Akpagana Koffi, PhD}

Laboratoire de Botanique et Ecologie végétale, Faculté des Sciences,

Université de Lomé, Lomé, Togo

Doi: 10.19044/esj.2017.v13n30p417 URL:http://dx.doi.org/10.19044/esj.2017.v13n30p417

\begin{abstract}
The Characterization of pollen content of Abomey-Calavi was studied from April 2015 to April 2017, whether two years. A floristic inventory of the species in 7 of the 12 districts of the municipality as well as a collection of pollen grains using the sensor of Hirst were carried out. In total, 104 species in bloom were inventoried each year. Pollen analysis yielded an average of 180 and 182 grains of pollen per cubic meter of air per day from April 2015 to April 2016 and April 2016 to April 2017. They belong to 29 families, 41 genera and 38 species. Among the species inventoried, $47.11 \%$ was found in the samples analyzed. The dominant taxa for the two years of study are: Acacia auriculiformis, Poaceae, Combretum indicum and Elaeis guineensis. The daily pollen index of these taxa shows that they are in bloom throughout the year and their main pollination period differs according to the seasons of the year. The correlation is positive between pollen grains and meteorological parameters such as maximum temperature and wind speed but negative between pollen grains, minimum temperature and rainfal.
\end{abstract}

Keywords: Characterization, pollen, meteorological parameters, floristic inventory, Abomey-Calavi 


\section{Résumé}

La caractérisation du contenu aéropollinique de la commune d'Abomey-Calavi a été étudiée d'avril 2015 à avril 2017, soit deux années. Un inventaire floristique des espèces dans 7 des 12 arrondissements de la commune ainsi qu'une collecte de grains de pollen à l'aide du capteur de Hirst ont été effectués. Au total, 104 espèces en fleurs ont été inventoriées chaque année. L'analyse pollinique a donné en moyenne par jour 180 et 182 grains de pollen par mètre cube d'air respectivement d'avril 2015 à avril 2016 et d'avril 2016 à avril 2017. Ils appartiennent à 29 familles, 41 genres et 38 espèces. Parmi les espèces inventoriées, une moyenne de $47,11 \%$ a été retrouvée dans les échantillons analysés. Les taxa dominants pour les deux années d'étude sont: Acacia auriculiformis, Poaceae, Combretum indicum et Elaeis guineensis. L'index pollinique journalier de ces taxa montre qu'ils sont en fleur pendant toute l'année et leur période principale de pollinisation diffère en fonction des saisons de l'année. La corrélation est positive entre les grains de pollen et les paramètres météorologiques tels que la température maximale et la vitesse du vent mais négative entre les grains de pollen, la température minimale et la pluviométrie.

Mots clés: Caractérisation, pollen, paramètres météorologiques, inventaire floristique, Abomey-Calavi

\section{Introduction}

Les allergies constituent la quatrième maladie dans le monde (Crossject Newletter, 2017). Au Bénin, selon le Ministère de la Santé, la prévalence des pathologies allergiques n'a cessé d'augmenter au cours des dernières décennies. En effet, les études épidémiologiques récentes soulignent l'augmentation rapide du nombre d'individus sujets à ces maladies allergiques. Les sources souvent évoquées sont les gaz des usines, des échappements des véhicules et des motos. Cependant, il existe dans l'air de nombreuses autres particules, notamment d'origine végétale, constituées par les pollens et les spores qui pourraient aussi être responsables de gênes respiratoires appelées pollinose de par leur forte concentration (Layachi, 2012). Dans la commune d'Abomey-Calavi, diverses espèces végétales existent à l'état naturel ou sont plantées le long des voies, dans les maisons et dans les espaces verts. Ces espèces émettent, au cours de leur floraison, d'énormes quantités de pollens dans l'atmosphère que l'aéropalynologie permet d'étudier (Bouziane et al., 2016) pour la réalisation de calendriers polliniques. La concentration de ces pollens dans l'air est sous l'influence des paramètres météorologiques (Clot, 2003). 
Au Bénin la première étude aéropalynologique a été réalisée sur le campus d'Abomey-Calavi par Tossou et al. (2016) avec l'appareil de Durham. Les travaux ont été étendus à toute la commune d'Abomey-Calavi au moyen du capteur de Hirst. L'objectif de la présente étude est de déterminer la charge pollinique de l'atmosphère de ladite commune en relation avec l'état de floraison des espèces et les paramètres météorologiques, d'avril 2015 à avril 2017.

\section{Milieu d'étude}

La commune d'Abomey Calavi est située au sud du Bénin entre $6^{\circ} 19^{\prime} 20^{\prime \prime}$ et $6^{\circ} 42^{\prime} 51^{\prime \prime}$ de latitude Nord et $2^{\circ} 11^{\prime} 45^{\prime \prime}$ et $2^{\circ} 25^{\prime} 15^{\prime \prime}$ de longitude Est. Elle couvre une superficie de 65000 ha pour une population de 307475 habitants (INSAE, 2013), soit une densité de $4,73 \mathrm{hab} / \mathrm{km}^{2}$.

Le climat au sud du Bénin est de type subéquatorial, marqué par deux saisons pluvieuses (avril à juillet et septembre à octobre) et deux saisons sèches (novembre à mars et août) (figure 1 et 2). Juin et juillet sont les mois les plus pluvieux en 2015 et ceux de mai et octobre en 2016. La quantité totale de pluies obtenues en 2015 est de 1360,5 mm d'eau et de $1236,4 \mathrm{~mm}$ d'eau en 2016. Les mois les plus secs sont janvier et décembre en 2015 et en 2016 avec respectivement $11,1 \mathrm{~mm}$ d'eau et $0 \mathrm{~mm}$ d'eau. La vitesse du vent dans la commune varie d'un mois à un autre. Elle est plus élevée en février $(7,61 \mathrm{~m} / \mathrm{s})$, mars $(7,24 \mathrm{~m} / \mathrm{s})$ et Janvier $(5,82 \mathrm{~m} / \mathrm{s})$ en 2015 et en août $(4,81$ $\mathrm{m} / \mathrm{s})$, avril $(4,74 \mathrm{~m} / \mathrm{s})$ et juillet $(4,74 \mathrm{~m} / \mathrm{s})$ en 2016 (figure 3)

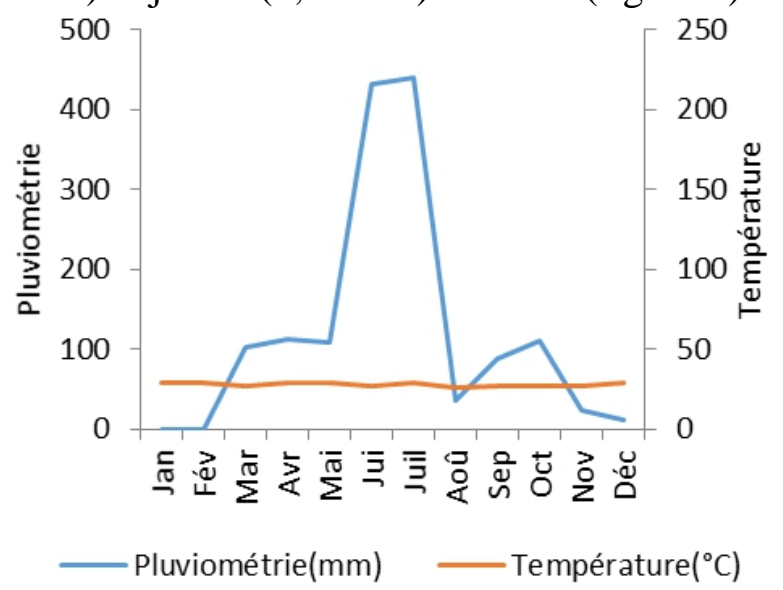

Figure 1 : Diagramme ombrothermique de la commune d'Abomey-Calavi au cours de l'année 2015 


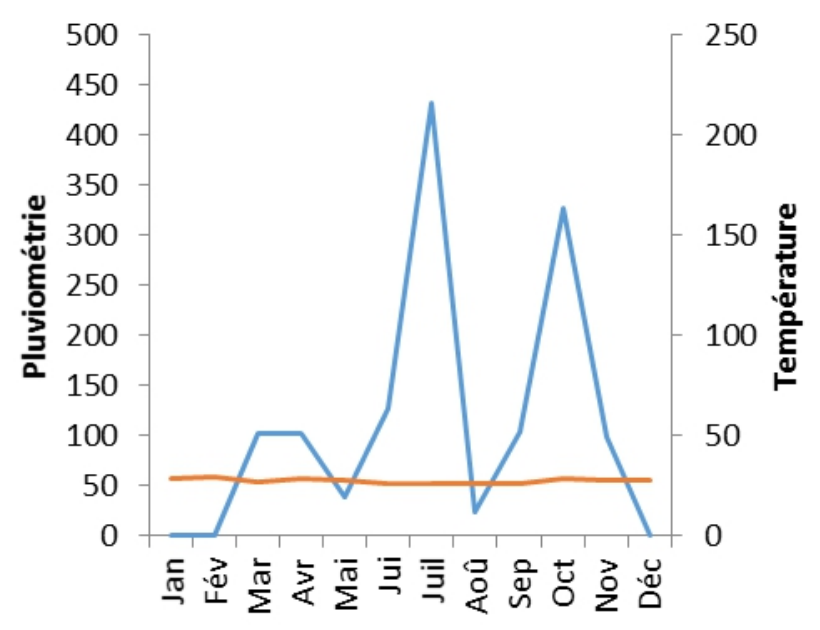

— Pluviométrie $(\mathrm{mm})$ Température $\left({ }^{\circ} \mathrm{C}\right)$

Figure 2 : Diagramme ombrothermique de la commune d'Abomey-Calavi au cours de l'année 2016

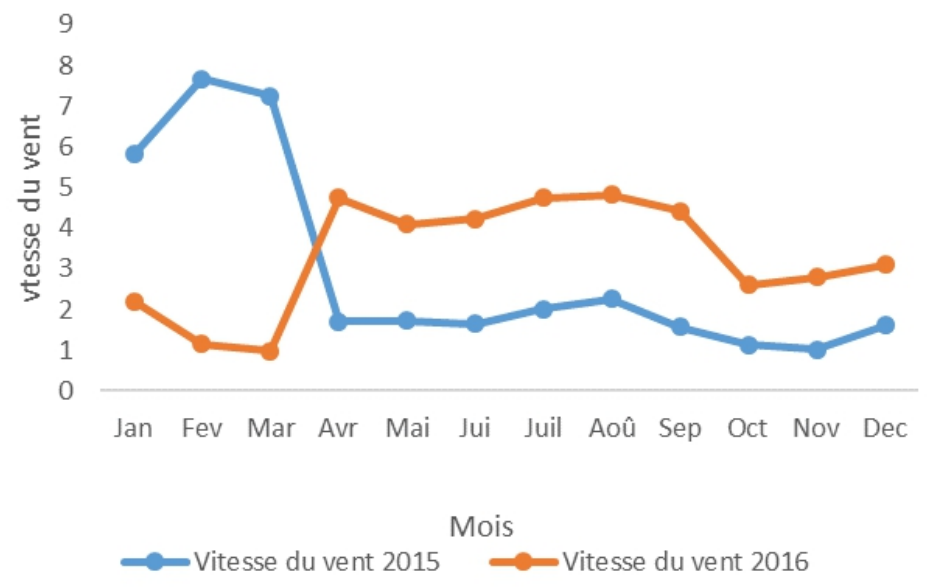

Figure 3 : courbes de la vitesse du vent au cours des années 2015 et 2016 


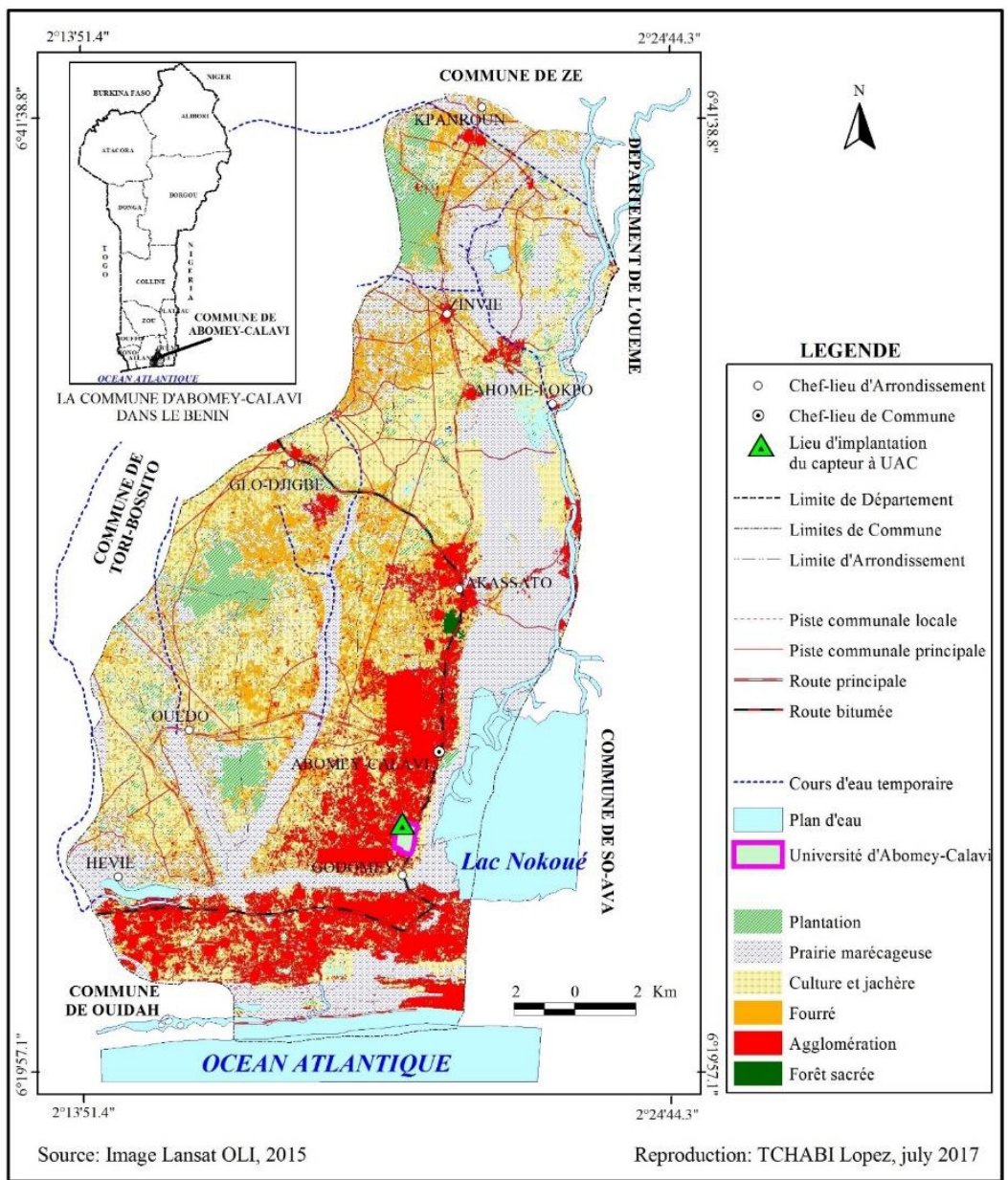

Figure 4 : Carte de la végétation de la commune d'Abomey-Calavi

La végétation de la commune d'Abomey-Calavi est caractéristique de celle du sud Bénin (Akoègninou, et al., 2006). Elle est composée d'un îlot de forêt, de fourrés, de mangrove, de plantations monospécifiques et de formations ouvertes comme les prairies marécageuses, les champs et jachères.

L'îlot de forêt est de type dense humide semi-décidue à Celtis spp (Akoègninou, 2004); il est sacré et mesure à peine un hectare de superficie. Il est situé sur un plateau de terre de barre. Les prairies marécageuses sont localisées dans les zones à hydromorphie permanente ou temporaire du bord du lac Nokoué et dans les bas-fonds de Godomey et de Calavi. Les plantations monospécifiques observées ça et là sont celles à Acacia auriculiformis et Elaeis guineensis. Les champs et jachères sont constitués des espèces fréquentes comme Zea mays, Celosia laxa, Lantana camara, etc. 


\section{Matériel et méthodes}

Un inventaire itinérant des espèces a été effectué dans 7 des 12 arrondissements que renferme la commune (Akassato, Ouèdo, Hêvié, Glodjigbé, Zinvié, Kpanroun et Togba). Celui des espèces en fleurs a été régulièrement réalisé chaque semaine à l'aide d'une fiche comportant la date du relevé, le nom de l'espèce et l'état de la floraison. Le logiciel google earth a été utilisé pour connaître les différentes zones de végétation (forêt dense, fourrées, plantations, prairie, cultures et jachères). La codification des espèces en fleurs a été effectuée suivant leur degré de floraison, 0 : absence de floraison ; 1 : début de floraison, 2 : maximum de floraison. Le premier relevé floristique a été effectué le 15 avril 2015.

L'échantillonnage aéropollinique a été réalisé de façon concomitante avec les relevés floristiques au moyen d'un capteur volumétrique de type Hirst placé à une hauteur de $13 \mathrm{~m}$ le 15 avril 2015 sur le toit du bâtiment de l'Herbier National du Bénin, sis à l'Université d'Abomey-Calavi. Ce système de captage permet l'aspiration de l'air à un débit constant de 10 litres d'air par minute. Les particules contenues dans l'air ont été piégées sur une bande adhésive transparente de cellophane enduite de résine et fixée sur un tambour animé d'un mouvement de rotation commandé par un système d'horlogerie qui défile devant la buse d'aspiration. Après sept (7) jours de fonctionnement, le tambour est retiré et remplacé par un autre sur lequel une autre bande de cellophane a été déjà fixée au laboratoire et protégée dans une boîte. La bande de cellophane retirée a été découpée en sept segments correspondant chacun à un jour de capture. Chaque segment de bande a été coloré par la fuschine et monté entre lame et lamelle pour une analyse quantitative et qualitative au microscope optique de marque Olympus aux grossissements X200, X400 et X1000. Le comptage des grains de pollen a été réalisé par balayage de 4 transects horizontaux des lames tel qu'il a été recommandé par le Réseau Espagnol d'Aéorobiologie (REA) (Galán et al., 2007).

Les identifications des grains de pollen ont été effectuées par comparaison avec les collections des lames de références de l'unité palynologique du Laboratoire de Botanique et Ecologie Végétale de l'Université d'Abomey-Calavi et des ouvrages d'illustrations des pollens et des spores de Sowunmi (1995) et Ybert (1980). Les identifications botaniques des espèces et des pollens ont été spécifiques ou génériques ou seulement au niveau de la famille. La nomenclature utilisée a été celle de Akoègninou et al. (2006).

L'échelle de Jäger et al. (1996) qui stipule que les grains de pollen les plus dominants ont un pourcentage $\geq 2 \%$ a été utilisée pour déterminer les familles, les genres et les espèces des grains de pollen dominants. 
La concentration moyenne journalière a été exprimée en nombre de grains de pollen par mètre cube d'air et par jour. L'estimation du nombre moyen de grains de pollen par mètre cube d'air a été obtenue en multipliant le nombre de grains de pollen effectivement comptés par le facteur de conversion de Jorgensen (1967). Les données polliniques ont été analysées pour déterminer la période principale de pollinisation (PPP) en utilisant la méthode des $95 \%$. Le début de la PPP a été défini à partir du moment où les concentrations cumulées atteignent ou dépassent $2,5 \%$ du total annuel, et elle se termine quand ce cumul atteint 97,5\% de ce même total (Andersen, 1991 et Bouziane et $a l .$, ). La période de pré-pic a été considérée comme celle comprise entre le début de la saison pollinique principale et le jour du pic qui correspond à la valeur maximale de concentration pollinique. La période de post-pic a été celle comprise entre le jour juste après le pic et la fin de la pollinisation.

Les corrélations entre les concentrations polliniques moyennes journalières et les paramètres météorologiques ont été réalisées à l'aide du test de Spearman à $\mathrm{P} \leq 0,05$ et $\mathrm{P} \leq 0,01$ du logiciel $\mathrm{R}$. Les variables météorologiques utilisées sont les données journalières des températures minimale, moyenne et maximale; de la vitesse du vent et de la pluviométrie.

\section{Résultats}

\section{Etats de floraison des espèces inventoriées}

Au total, 104 espèces ont été inventoriées au cours de chaque année. Les plus fréquentes sont :

Mallotus oppositifolius, Mangifera indica, Senna siamea, Acacia auriculiformis, Albizia lebbeck, Andropogon gayanus, Cenchrus ciliaris, Panicum maximum, Combretum indicum, Carica papaya, Chassalia kolly, Cnestis ferruginea, Elaeis guineensis, Fluggea virosa, Jatropha curcas, Cenchrus ciliaris, Melia azadirachta, Cocos nucifera, etc.

La figure 5 donne l'évolution des différents états de floraison des espèces inventoriées d'avril 2015 à avril 2017. 


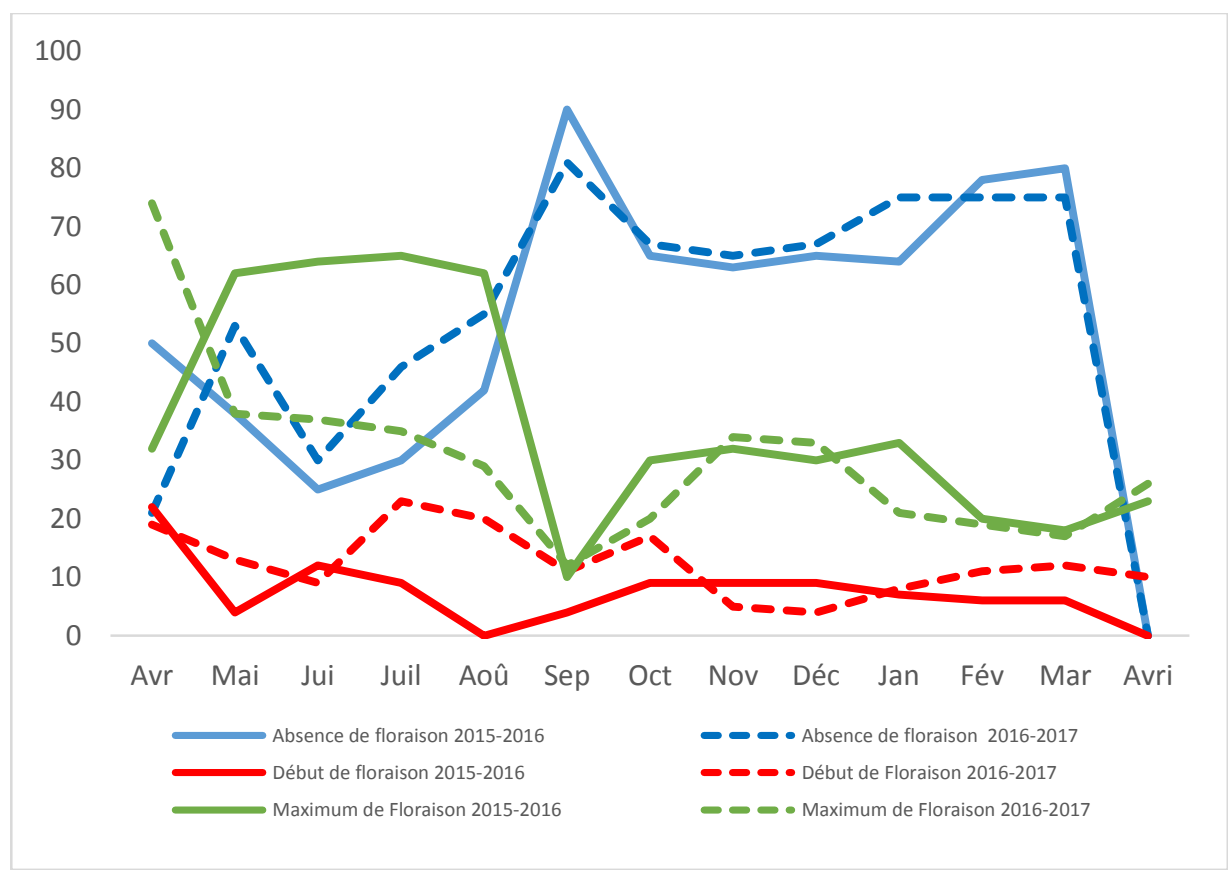

Figure 5 : Etats de floraison des espèces inventoriées de 2015 à 2017

Les courbes d'états de floraison des espèces inventoriées au cours des deux années sont similaires. Les espèces non en fleurs sont en faible nombre au cours des mois d'avril à août (grande saison pluvieuse et petite saison sèche) avec un maximum de 50 espèces ; leur nombre croît au milieu de la petite saison sèche et atteint un pic de 90 espèces en septembre (début de la petite saison pluvieuse). Ce nombre diminue progressivement en octobre et reste constant jusqu'au mois de janvier où il connaît un léger accroissement jusqu'en mars puis chute brutalement en avril. La même tendance est obtenue au cours de la deuxième année.

Les espèces en début de floraison sont en nombre élevé dans les mois de mai, juin, juillet et août (grande saison pluvieuse et petite saison sèche) et restent en nombre constant de septembre à avril (fin des pluies et grande saison sèche)

Les espèces en maximum de floraison sont abondantes au cours des mois pluvieux (avril à juillet), rares au cours du mois d'août et au cours de la petite saison pluvieuse (septembre à octobre). Leur nombre croît légèrement en octobre puis décroît à partir du mois de janvier.

\section{Spectre pollinique}

$\mathrm{Au}$ total, 66109 grains de pollen sont identifiés au cours de la première année et sont répartis en quarante-cinq (45) taxa appartenant à 29 familles, 41 genres et 38 espèces. La seconde année enregistre le même 
nombre de taxa, de familles et de genres mais le nombre de grains de pollen (66870) est légèrement supérieur à celui de la première année (tableau 1 , annexe).

Les taxa dominants en nombre de grains de pollen au cours de la première et de la deuxième année se présentent respectivement comme suit: Acacia auriculiformis: 18407 (27,84\%) et 17979 (26,88\%); Poaceae: 13356 $(20,2 \%)$ et $14182(21,2 \%)$; Combretum indicum : $9415(14,24 \%)$ et 9498 (14,20\%) et enfin Elaeis guineensis avec 4041 (6,11\%) et 4192 (6,26\%.).

Les mois les plus riches en grains de pollen au cours de la première année sont: mai avec 6977 , soit $10,84 \%$; juin avec 6412 , soit $9,96 \%$; janvier avec 6267 , soit $9,74 \%$ et août qui totalise 5372 grains de pollen, soit $8,35 \%$. Les mois ayant enregistré les plus faibles nombres de grains de pollen sont : octobre avec $4246(6,60 \%)$, novembre avec $4258(6,62 \%)$ et mars qui fait un total de 4792 grains de pollen, soit 7,45\%. En 2016-2017, les mois les plus riches en grains de pollen sont avril avec 9094, soit 13,06\% ; mai avec 6612, soit 29,49\%; juin avec 6608, soit 9,49\% et décembre avec 6465 , soit $9,28 \%$. Les mois les plus pauvres en grains de pollen sont janvier, novembre, et octobre avec respectivement 3700, soit 5,31\%, 3903, soit $5,60 \%$ et 4145 , soit $5,95 \%$.

Les mois les plus riches en nombre de grains de pollen au cours des deux années (avril, mai, juin, août, janvier et décembre) appartiennent à la grande saison des pluies, à la petite saison sèche et à la grande saison sèche. De même, les mois les moins riches en grains de pollen (janvier, novembre, octobre) sont de la grande saison sèche et de la petite saison des pluies.

\section{Variation saisonnière des grains de pollen}

La concentration des grains de pollen dans l'atmosphère varie au cours des différentes saisons d'une année (figure 6).

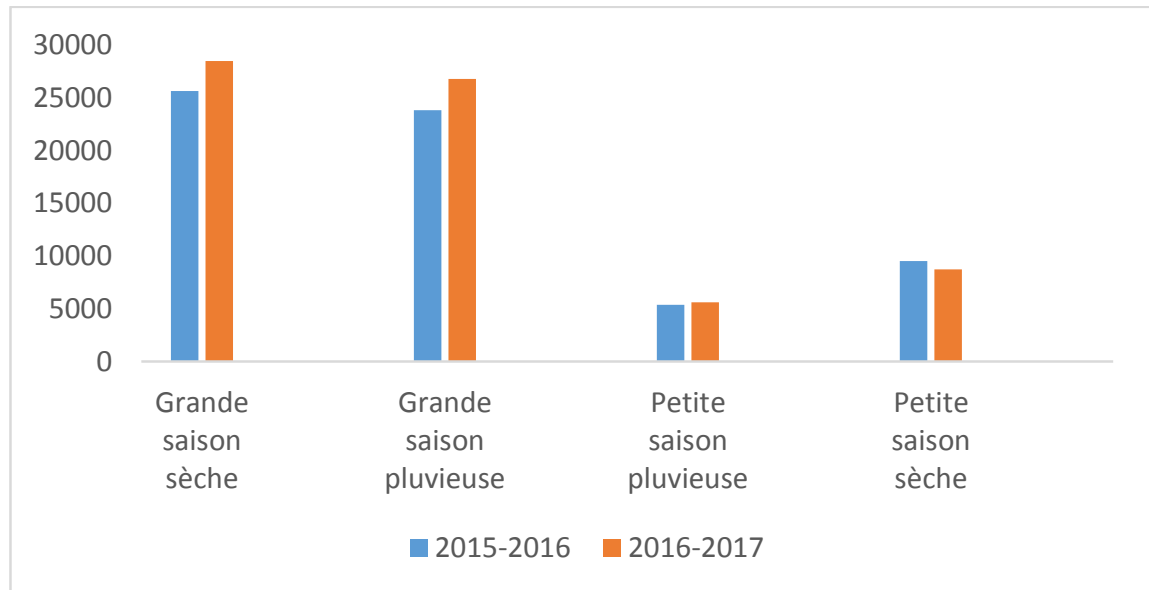

Figure 6 : Variation du nombre de grains de pollen en fonction des saisons de l'année 
Au cours des deux années, ce sont les grandes sèches et les grandes saisons pluvieuses qui ont enregistré les plus grands nombres de grains de pollen (figure 6). Les petites saisons sèches et les petites saisons pluvieuses ont par contre connu les plus faibles nombres.

Le tableau 2 présente les taxa de pollens les plus dominants au cours des différentes saisons. Il s'agit de: Acacia auriculiformis, Poaceae, Combretum indicum et Elaeis guinneensis.

Tableau 2: Variation du nombre des grains de pollen des espèces dominantes au cours des différentes saisons.

\begin{tabular}{|c|c|c|c|c|c|c|c|c|}
\hline Année & \multicolumn{4}{|c|}{$2015-2016$} & \multicolumn{5}{c|}{$2016-2017$} \\
\hline Saisons & GSS & GSP & PSS & PSP & GSS & GSP & PSS & PSP \\
\hline $\begin{array}{c}\text { Acacia } \\
\text { auriculiformis, }\end{array}$ & 8954 & 6004 & 1198 & 2251 & 7839 & 6341 & 1944 & 1855 \\
\hline Poaceae, & 4889 & 5408 & 917 & 2142 & 5084 & 5956 & 1032 & 2110 \\
\hline Combretum indicum & 3765 & 3460 & 645 & 1545 & 4139 & 2701 & 1143 & 1515 \\
\hline Elaeis guinneensis. & 1940 & 1108 & 341 & 652 & 1910 & 1342 & 287 & 651 \\
\hline
\end{tabular}

GSS : Grande Saison Sèche, GSP : Grande Saison Pluvieuse, PSS : Petite Saison Sèche, PSP : Petite Saison pluvieuse

\section{Evolution journalière et période principale de pollinisation des taxa de pollens dominants}

Les figures 7 à 14 présentent l'évolution journalière en grains de pollen des espèces les plus dominantes.

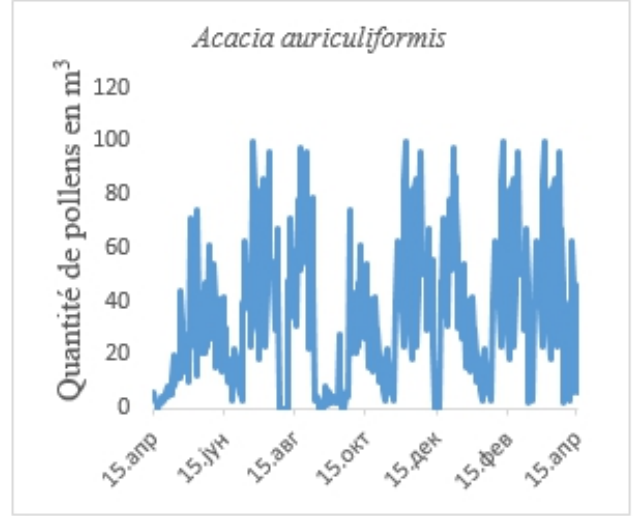

Figure 7: Evolution journalière de Acacia auriculiformis en 2015-2016

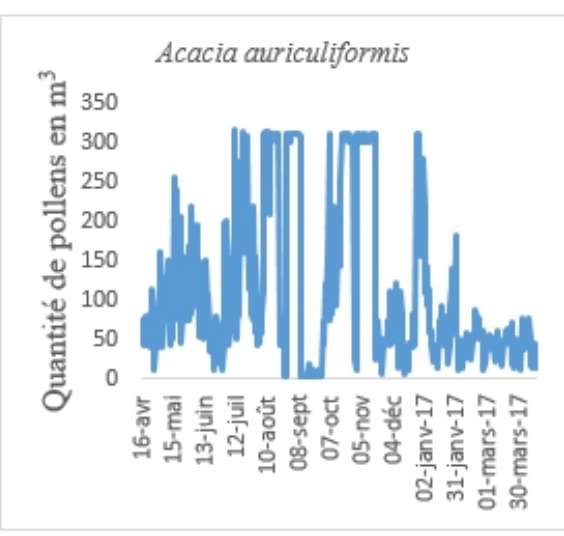

Figure \&: Evolution journalière de Acacia auriculiformis en 2016-2017 


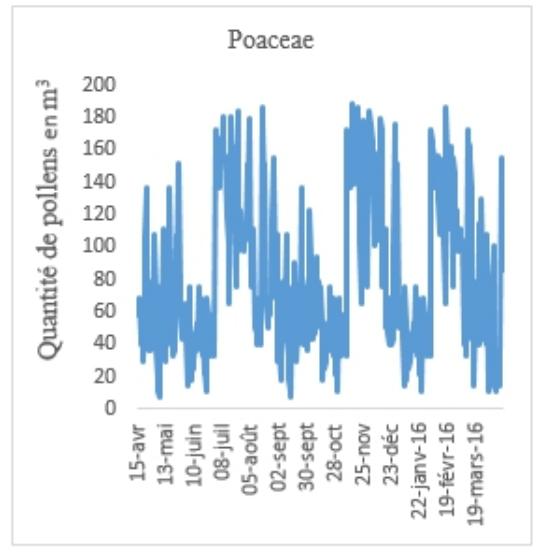

Figure 9: Evolution journalière des Poaceae en 2015-2016

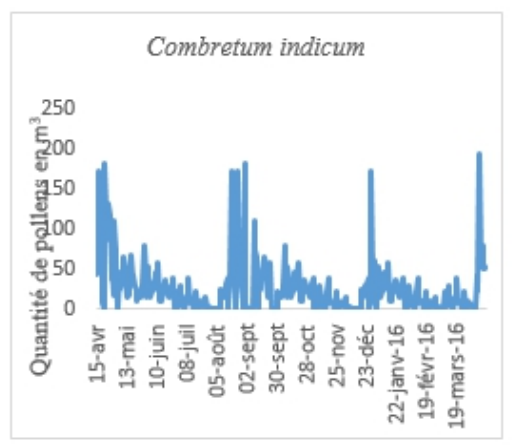

Figure 11 : Evolution journalière de Combretum indicum en 2015-2016

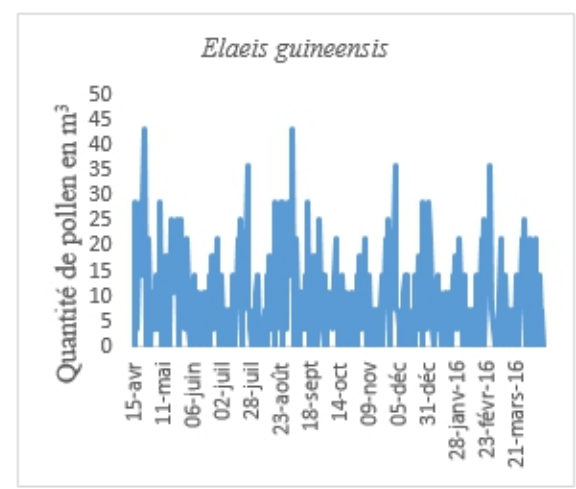

Figure 13: Evolution des pollens de Elaeis guineensis en $2015-2016$

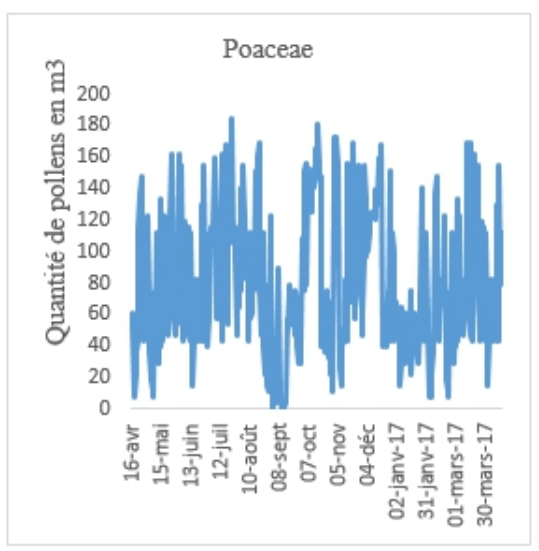

Figure 10: Evolution journalière des Poaceae en 2016-2017

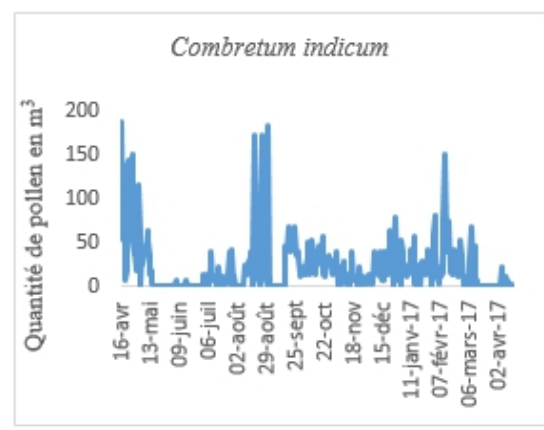

Figure 12 : Evolution journalère de Combretum indicum en 2016-2017

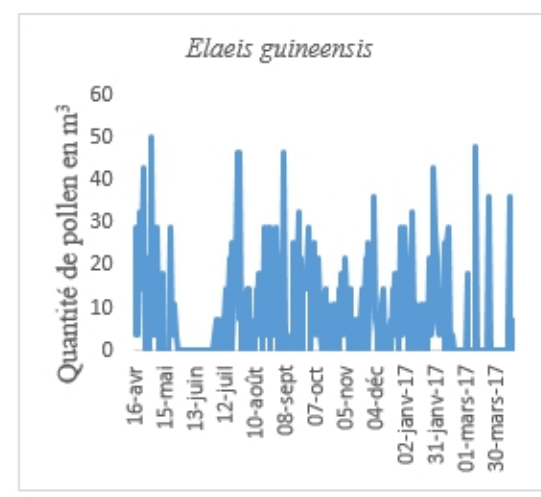

Figure 14: Evolution journalière des pollens de Elaeis guineensis en 2016-2017

Les graphes d'évolution journalière montrent des groupes de pics de concentration pollinique. Au cours de la première année, 8 groupes de pics de concentration sont distingués pour les pollens de Acacia auriculiformis 4 
groupes de pics pour les Poaceae et les pollens de Combretum indicum et 6 groupes de pics pour les grains de pollen d'Elaeis guineensis.

$\mathrm{Au}$ cours de la deuxième année, les pollens de Acacia auriculiformis présentent 5 groupes de pics de concentration, les Poaceae en présentent 4, Combretum indicum seulement 3 et Elaeis guineensis, 6.

Il ressort de ces graphes que d'une année à une autre, le nombre de groupes de concentration en grains de pollen est stable pour les Poaceae et Elaeis guineensis, ce qui n'est pas le cas chez

\section{Acacia auriculiformis et Combretum indicum.}

La présence permanente des espèces dominantes dans l'atmosphère, ne permet pas de déterminer leur Période Principale de Pollinisation (PPP) sur une année. Il a fallu recourir aux différentes saisons de l'année. Les résultats obtenus sont consignés dans le tableau 3.

Tableau 3: Paramètres de la période principale de pollinisation (PPP) des taxa dominants suivant les saisons de l'année.

\begin{tabular}{|c|c|c|c|c|c|c|c|}
\hline \multicolumn{8}{|c|}{ Grande saison pluvieuse } \\
\hline taxa & Années & $\begin{array}{c}\text { Date initiale } \\
\text { de PPP }\end{array}$ & $\begin{array}{c}\text { Date } \\
\text { finale } \\
\text { de PPP }\end{array}$ & $\begin{array}{l}\text { Durée } \\
\text { en } \\
\text { jour }\end{array}$ & $\begin{array}{c}\text { Total } \\
\text { grains de } \\
\text { pollen } / \mathrm{m}^{3} \\
\text { d'air de } \\
\text { PPP }\end{array}$ & $\begin{array}{c}\text { Date du pic } \\
\text { de } \\
\text { concentration }\end{array}$ & $\begin{array}{l}\text { Concentration } \\
\text { maximale }\end{array}$ \\
\hline \multirow[t]{2}{*}{$\begin{array}{c}\text { Acacia } \\
\text { auriculiformis }\end{array}$} & $\begin{array}{l}2015- \\
2016\end{array}$ & 2 mai 2015 & $\begin{array}{c}30 \\
\text { juillet } \\
2015\end{array}$ & 89 & 11718 & $\begin{array}{c}14 \text { juillet } \\
2015\end{array}$ & 346 \\
\hline & $\begin{array}{l}2016- \\
2017\end{array}$ & $\begin{array}{l}19 \text { avril } \\
2016\end{array}$ & $\begin{array}{c}30 \\
\text { juillet } \\
2016\end{array}$ & 102 & 11437 & $\begin{array}{l}20 \text { juillet } \\
2016\end{array}$ & 313 \\
\hline \multirow[t]{2}{*}{ Poaceae } & $\begin{array}{l}2015- \\
2016\end{array}$ & $\begin{array}{l}19 \text { avril } \\
2015\end{array}$ & $\begin{array}{c}29 \\
\text { juillet } \\
2015\end{array}$ & 101 & 9389 & $\begin{array}{l}15 \text { juillet } \\
2015\end{array}$ & 360 \\
\hline & $\begin{array}{l}2016- \\
2017\end{array}$ & $\begin{array}{l}15 \text { avril } \\
2016\end{array}$ & $\begin{array}{c}26 \\
\text { juillet } \\
2016\end{array}$ & 102 & 9749 & $\begin{array}{l}13 \text { juillet } \\
2016\end{array}$ & 439 \\
\hline \multirow[t]{2}{*}{$\begin{array}{l}\text { Combretum } \\
\text { indicum }\end{array}$} & $\begin{array}{l}2015- \\
2016\end{array}$ & 15avril 2015 & $\begin{array}{c}17 \\
\text { juillet } \\
2015 \\
\end{array}$ & 106 & 3391 & $1^{\mathrm{er}}$ mai 2015 & 112 \\
\hline & $\begin{array}{l}2016- \\
2017\end{array}$ & $1^{\mathrm{er}}$ avril 2016 & $\begin{array}{c}28 \text { mai } \\
2016\end{array}$ & 57 & 2452 & $1^{\mathrm{er}}$ mai 2016 & 194 \\
\hline \multirow[t]{2}{*}{$\begin{array}{c}\text { Elaeis } \\
\text { guineensis }\end{array}$} & $\begin{array}{l}2015- \\
2016\end{array}$ & $\begin{array}{l}15 \text { avril } \\
2015\end{array}$ & $\begin{array}{c}26 \\
\text { juillet } \\
2015 \\
\end{array}$ & 101 & 1015 & 23 avril 2015 & 43 \\
\hline & $\begin{array}{l}2016- \\
2017\end{array}$ & $\begin{array}{c}17 \text { avril } \\
2016\end{array}$ & $\begin{array}{c}30 \\
\text { juillet } \\
2016\end{array}$ & 104 & 781 & 15 juin 2016 & 49 \\
\hline \multicolumn{8}{|c|}{ Petite saison pluvieuse } \\
\hline
\end{tabular}




\begin{tabular}{|c|c|c|c|c|c|c|c|}
\hline \multirow[t]{2}{*}{$\begin{array}{c}\text { Acacia } \\
\text { auriculiformis }\end{array}$} & $\begin{array}{l}2015- \\
2016\end{array}$ & $\begin{array}{l}3 \text { octobre } \\
2015\end{array}$ & $\begin{array}{c}27 \\
\text { octobre } \\
2015\end{array}$ & 24 & 1728 & $\begin{array}{l}27 \text { octobre } \\
2015\end{array}$ & 144 \\
\hline & $\begin{array}{l}2016- \\
2017\end{array}$ & $\begin{array}{l}10 \text { octobre } \\
2016\end{array}$ & $\begin{array}{c}27 \\
\text { octobre } \\
2016\end{array}$ & 17 & 2117 & $\begin{array}{l}08 \text { octobre } \\
2016\end{array}$ & 220 \\
\hline \multirow[t]{2}{*}{ Poaceae } & $\begin{array}{l}2015- \\
2016\end{array}$ & $\begin{array}{c}05 \\
\text { septembre } \\
2015\end{array}$ & $\begin{array}{c}24 \\
\text { octobre } \\
2015\end{array}$ & 49 & 2804 & $\begin{array}{c}\text { 08 septembre } \\
2015\end{array}$ & 137 \\
\hline & $\begin{array}{l}2016- \\
2017\end{array}$ & $\begin{array}{c}04 \\
\text { septembre } \\
2016\end{array}$ & $\begin{array}{c}28 \\
\text { octobre } \\
2016\end{array}$ & 54 & 2768 & $\begin{array}{l}26 \text { septembre } \\
2016\end{array}$ & 202 \\
\hline \multirow[t]{2}{*}{$\begin{array}{l}\text { Combretum } \\
\text { indicum }\end{array}$} & $\begin{array}{l}2015- \\
2016\end{array}$ & $\begin{array}{c}4 \text { septembre } \\
2015\end{array}$ & $\begin{array}{c}27 \\
\text { octobre } \\
2015\end{array}$ & 53 & 1508 & $\begin{array}{c}09 \text { septembre } \\
2015\end{array}$ & 122 \\
\hline & $\begin{array}{l}2016- \\
2017\end{array}$ & $\begin{array}{c}10 \\
\text { septembre } \\
2016\end{array}$ & $\begin{array}{c}27 \\
\text { octobre } \\
2016\end{array}$ & 47 & 1400 & $\begin{array}{c}18 \text { septembre } \\
2016\end{array}$ & 202 \\
\hline \multirow[t]{2}{*}{$\begin{array}{c}\text { Elaeis } \\
\text { guineensis }\end{array}$} & $\begin{array}{l}2015- \\
2016\end{array}$ & $\begin{array}{c}3 \text { septembre } \\
2015\end{array}$ & $\begin{array}{c}27 \\
\text { octobre } \\
2015\end{array}$ & 54 & 418 & $\begin{array}{c}24 \text { septembre } \\
2015\end{array}$ & 43 \\
\hline & $\begin{array}{l}2016- \\
2017\end{array}$ & $\begin{array}{c}4 \text { septembre } \\
2016\end{array}$ & $\begin{array}{c}26 \\
\text { octobre } \\
2016 \\
\end{array}$ & 52 & 508 & $\begin{array}{l}09 \text { octobre } \\
2016\end{array}$ & 32 \\
\hline \multicolumn{8}{|c|}{ Grande saison sèche } \\
\hline \multirow[t]{2}{*}{$\begin{array}{c}\text { Acacia } \\
\text { auriculiformis }\end{array}$} & $\begin{array}{l}2015- \\
2016\end{array}$ & $\begin{array}{c}3 \text { novembre } \\
2015\end{array}$ & $\begin{array}{c}25 \text { avril } \\
2016\end{array}$ & 163 & 21971 & - & \\
\hline & $\begin{array}{l}2016- \\
2017\end{array}$ & $\begin{array}{c}5 \text { novembre } \\
2016\end{array}$ & $\begin{array}{c}13 \text { avril } \\
2017\end{array}$ & 147 & 21067 & - & \\
\hline \multirow[t]{2}{*}{ Poaceae } & $\begin{array}{l}2015- \\
2016 \\
\end{array}$ & $\begin{array}{c}9 \text { novembre } \\
2015 \\
\end{array}$ & $\begin{array}{c}23 \text { mars } \\
2016\end{array}$ & 133 & 16247 & - & \\
\hline & $\begin{array}{l}2016- \\
2017 \\
\end{array}$ & $\begin{array}{c}9 \text { novembre } \\
2016 \\
\end{array}$ & $\begin{array}{c}23 \text { mars } \\
2017 \\
\end{array}$ & 123 & 17318 & - & \\
\hline \multirow[t]{2}{*}{$\begin{array}{c}\text { Combretum } \\
\text { indicum }\end{array}$} & $\begin{array}{l}2015- \\
2016\end{array}$ & $\begin{array}{c}1^{\text {er Novembre }} \\
2015\end{array}$ & $\begin{array}{c}1^{\text {er Avril, }} \\
2016\end{array}$ & 150 & 2210 & $\begin{array}{c}1^{\text {er Avril, }} \\
2016\end{array}$ & 194 \\
\hline & $\begin{array}{l}2016- \\
2017 \\
\end{array}$ & $\begin{array}{c}1^{\text {er Novembre }} \\
2016\end{array}$ & $\begin{array}{c}1^{\text {er Avril, }} \\
2017\end{array}$ & 150 & 2128 & $\begin{array}{c}1^{\text {er Avrill, }} \\
2017\end{array}$ & 203 \\
\hline \multirow[t]{2}{*}{$\begin{array}{c}\text { Elaeis } \\
\text { guineensis }\end{array}$} & $\begin{array}{l}2015- \\
2016\end{array}$ & $\begin{array}{c}06 \\
\text { novembre } \\
2015 \\
\end{array}$ & $\begin{array}{c}31 \text { mars } \\
2015\end{array}$ & 144 & 1188 & & \\
\hline & $\begin{array}{l}2016- \\
2017\end{array}$ & $\begin{array}{c}10 \\
\text { novembre } \\
2016 \\
\end{array}$ & $\begin{array}{c}27 \text { mars } \\
2017\end{array}$ & 136 & 1089 & & \\
\hline \multicolumn{8}{|c|}{ Petite saison sèche } \\
\hline \multirow[t]{2}{*}{$\begin{array}{c}\text { Acacia } \\
\text { auriculiformis }\end{array}$} & $\begin{array}{l}2015- \\
2016 \\
\end{array}$ & 2 août 2015 & $\begin{array}{c}29 \text { août } \\
2015\end{array}$ & 27 & 4734 & 29 août 2015 & 313 \\
\hline & $\begin{array}{l}2016- \\
2017 \\
\end{array}$ & 2 août 2016 & $\begin{array}{c}29 \text { août } \\
2016\end{array}$ & 27 & 1897 & 11 août 2016 & 223 \\
\hline Poaceae & $2015-$ & 3 août 2015 & 29 août & 26 & 2617 & 27 août 2015 & 314 \\
\hline
\end{tabular}




\begin{tabular}{|c|c|c|c|c|c|c|c|}
\hline & 2016 & & 2015 & & & & \\
\hline & $\begin{array}{l}2016- \\
2017\end{array}$ & 3 août 2016 & $\begin{array}{l}29 \text { août } \\
2016\end{array}$ & 26 & 2333 & 16 août 2016 & 321 \\
\hline \multirow[t]{2}{*}{$\begin{array}{c}\text { Combretum } \\
\text { indicum }\end{array}$} & $\begin{array}{l}2015- \\
2016\end{array}$ & 05 août 2015 & $\begin{array}{l}30 \text { août } \\
2015\end{array}$ & 25 & 961 & 17 août 2015 & 184 \\
\hline & $\begin{array}{l}2016- \\
2017\end{array}$ & 08 août 2016 & $\begin{array}{c}28 \text { août } \\
2016\end{array}$ & 20 & 954 & 22 août 2016 & 184 \\
\hline \multirow[t]{2}{*}{$\begin{array}{c}\text { Elaeis } \\
\text { guineensis }\end{array}$} & $\begin{array}{l}2015- \\
2016 \\
\end{array}$ & 2 août 2015 & $\begin{array}{c}29 \text { août } \\
2015\end{array}$ & 27 & 274 & 22 août 2015 & 41 \\
\hline & $\begin{array}{l}2016- \\
2017\end{array}$ & 03 août 2016 & $\begin{array}{c}27 \text { août } \\
2016\end{array}$ & 24 & 227 & 10 août 2016 & 43 \\
\hline
\end{tabular}

Les PPP des espèces dominantes au cours des grandes saisons pluvieuses s'étendent d'avril à juillet sauf pour Acacia auriculiformis où elle débute en mai en 2015. Au cours des petites saisons pluvieuses elles se situent entre septembre et octobre exceptée pour Acacia auriculiformis où elle se situe en octobre. Leur PPP au cours des grandes saisons sèches débute en novembre et se termine pour certaines en mars et pour les autres en avril. Quant aux petites saisons sèches, la PPP se situe entre le début et la fin du mois d'août. Les pics polliniques et les concentrations maximales des espèces dominantes varient d'une espèce à une autre et d'une saison à une autre. Ils se situent en grande saison pluvieuse en avril, mai, juin et juillet. En petite saison pluvieuse, ils sont observés entre septembre et octobre et en août au cours de la petite saison sèche. Au cours de la grande saison sèche, le pic pollinique a lieu en avril mais seulement pour Combretum indicum. Les concentrations polliniques maximales les plus élevées, obtenues sont de 439 en 2016-2017 et de 360 en 2015-2016 pour la même espèce. Les plus faibles valeurs enregistrées sont de 32 grains de pollen au cours de la petite saison pluvieuse de 2015-2016 et de 41 grains au cours de la petite saison sèche de 2015-2016.

\section{Corrélation entre les concentrations journalières des grains de pollen et les facteurs météorologiques}

Le tableau 4 présente les résultats de corrélation entre les concentrations polliniques journalières et les paramètres météorologiques

Tableau 4: Test de Corrélation de Spearman entre les quantités journalières de grains de pollen et les données journalières des paramètres météorologiques

\begin{tabular}{|c|c|c|c|}
\hline $\begin{array}{c}\text { Paramètres } \\
\text { météorologiques }\end{array}$ & Années & $\begin{array}{l}\text { Valeurs de } \\
\text { corrélation }\end{array}$ & $\begin{array}{l}\text { Valeurs de la } \\
\text { probabilité }\end{array}$ \\
\hline Température maximale & $\begin{array}{l}2015- \\
2016\end{array}$ & 0,175 & 0,003 \\
\hline & $\begin{array}{l}2016- \\
2017\end{array}$ & 0,23 & 0,01 \\
\hline Température minimale & $\begin{array}{l}2015- \\
2016\end{array}$ & $-0,148$ & 0,0005 \\
\hline
\end{tabular}




\begin{tabular}{cccc}
\hline \hline \multirow{3}{*}{ Température moyenne } & $2016-$ & $-0,156$ & 0,0004 \\
& 2017 & & \\
& $2015-$ & 0,174 & 0,004 \\
& 2016 & & 0,002 \\
& $2016-$ & 0,177 & 0,0006 \\
\multirow{3}{*}{ Vitesse du vent } & 2017 & & 0,0002 \\
& $2015-$ & 0,151 & 0,001 \\
& 2016 & & \\
& $2016-$ & 0,158 & 0,0001 \\
\hline \multirow{2}{*}{ Pluviométrie } & 2017 & $-0,78$ & \\
& $2015-$ & $-0,69$ & \\
& 2016 & & \\
& $2016-$ & &
\end{tabular}

Il ressort du tableau que toutes les variables météorologiques considérées exercent une influence sur la dispersion des grains de pollen dans l'atmosphère. Les valeurs de probabilité obtenues avec chacune des variables sont inférieures à 0,05 . Au cours des deux années d'étude, on constate une corrélation négative entre la température minimale, la pluviométrie et la quantité de pollens dans l'atmosphère. La quantité de pollens diminue quand la pluie augmente et quand la température baisse. Cette corrélation est positive entre les températures moyennes et maximales, la vitesse du vent et la quantité de grains de pollen. Le nombre de grains de pollen augmente dans l'atmosphère quand la vitesse du vent et la température sont élevées.

\section{Discussion}

Les espèces en fleurs inventoriées d'avril 2015 à avril 2016 et d'avril 2016 à avril 2017 ont une représentativité moyenne de 47,11\%. Elle concerne moins de la moitié des espèces inventoriées. La comparaison mensuelle entre les espèces en fleurs et la pluie pollinique montre que la quantité de grains de pollen présents dans l'atmosphère augmente en fonction du nombre d'espèces en floraison. En effet d'avril 2015 à avril 2016, les mois les plus riches en pollen sont mai, juin, janvier et août (tableau 1) et les résultats du relevé floristique effectué (figure 5) indiquent que ces mois plus riches en grains de pollen ont connu un maximum d'espèces en début ou en maximum de floraison. Ces résultats se confirment au cours de la seconde année de l'étude ou les mois les plus riches en grains de pollen (avril, mai et juin) ont enregistré sur le plan floristique le maximum d'espèces en pleine floraison. Ces résultats permettent de dire que la quantité de grains de pollen dispersés dans l'atmosphère dépend de l'état de floraison des espèces (Kasprzyk, 2003 et Thibaudon et Lachasse, 2005) 
Le nombre total de grains de pollens enregistrés au cours des deux années d'étude est de 132979. D'avril 2015 à avril 2016, 66109 grains de pollen répartis en 29 familles, 41 genres et 38 espèces avec une concentration journalière de 180 grains de pollen par mètre cube d'air ont été identifiés. D’avril 2016 à avril 2017, 66870 grains de pollen ont été comptés, soit 182 grains de pollen par mètre cube d'air par jour. Ces valeurs sont plus de 6 fois élevées que celles obtenues par Tossou et al. (2016) qui ont eu seulement 5053 grains de pollen dans l'atmosphère du campus d'AbomeyCalavi pendant une année avec l'appareil de Durham. Cette grande différence observée serait due au type d'appareil utilisé pour la capture des grains de pollen. La quantité de pollens par volume d'air (180 grains par volume d'air d'avril 2015 à avril 2016 et 182 grains d'avril 2016 à avril 2017) obtenue au cours de cette étude reste plus élevée que celle enregistrée par Manju et Swati (2008) qui ont obtenu 3 grains de pollen $/ \mathrm{m}^{3}$ en Inde, de Hawke et al. (1989) qui ont eu 12 grains de pollen $/ \mathrm{m}^{3}$ en Afrique du Sud mais elle reste faible par rapport à celle obtenue par Fabiana et Claudio (1997) avec 330 grains de pollens $/ \mathrm{m}^{3}$ d'air en Argentine avec l'appareil de Hirst. Ces variations observées pourraient s'expliquer par le type de végétation de chaque région, la phénologie des espèces, les conditions météorologiques du milieu, la position géographique et les rythmes de dispersion (Essien, 2014).

La différence quantitative en grains de pollen observée entre les deux années d'étude pourrait s'expliquer par le retard accusé par les premières pluies au cours de la deuxième année d'étude. Selon Clot (2003), les retards de précipitations peuvent entraîner des périodes polliniques très longues et des pics élevés. Parmi les taxa de pollens identifiés, ceux de Acacia auriculiformis et des Poaceae sont les plus abondants au cours des différentes saisons de l'année. Ceci serait dû à la présence de nombreux pieds de l'espèce à proximité du site d'expérimentation, dans les différentes localités du milieu d'étude et de la plantation de l'espèce à environ 150 mètres du site d'implantation du capteur. Quant aux Poaceae cela s'expliquerait par la présence au cours de la saison des pluies de nombreux champs de maïs et notamment de Panicum maximum très répandue dans la zone guinéenne.

La présence des grains de pollen de Ceiba pentadra (Bombacaceae), non recensée dans la végétation locale, indique que certaines espèces de la végétation lointaine peuvent être enregistrées grâce à la capacité de transport de leurs grains de pollen par le vent sur de grande distance. Selon Clot (2003), les grains de pollen sont capables de voyager sur une distance de $2500 \mathrm{~km}$.

D’un point de vue phénologique, les caractéristiques des périodes principales de pollinisation (PPP) sont variables d'une année à l'autre. La 
pollinisation, fortement influencée par les facteurs météorologiques, peut être précoce ou tardive. Sa durée peut varier; suivant les saisons de l'année et être courte ou longue (Achmakh et al., 2006). Les espèces dominantes: Acacia auriculiformis, Poaceae, Combretum indicum, Elaeis guineensis ont une période principale de pollinisation au cours de chaque saison de l'année. Leurs courbes d'évolution journalière (figures 7 à 14) montrent que ces espèces sont présentes et restent en fleurs pendant toute l'année. Ainsi, il apparait qu'elles ont une période principale de pollinisation pendant les mêmes saisons des deux années. Ces résultats sont contraires à ceux de Bouziane et al. (2016), Trigo et al. (1999) et de Garcia et al. (1997) qui ont pu déterminer dans leurs études respectives à Tétouan (Maroc) et en Espagne une période principale de pollinisation de chaque espèce pendant toute une année. Ceci pourrait s'expliquer par le type de climat de chaque région, la différence des végétations et la phénologie des espèces de ces végétations.

Les variations des quantités de pollens capturés d'un mois à l'autre seraient dues aux conditions météorologiques dont principalement la température, la pluviométrie et la vitesse du vent (Hyunen et al., 2003). Les tests de corrélations effectués ont montré qu'il existe une corrélation négative entre la température minimale et les grains de pollens présents dans l'atmosphère corroborant les résultats de Green et al. (2004) et de Bouziane et $a l$. (2016) qui ont trouvé respectivement à Tétouan (Maroc) et à Màlaga (Espagne) que les grains de pollen ne sont pas nombreux dans l'atmosphère quand la température est basse. Selon ces auteurs une température basse rend l'atmosphère lourde et empêche le maintien constant des grains de pollen dans l'air. La corrélation négative obtenue entre les concentrations polliniques et la pluviométrie indique que la pollinisation diminue lorsque les précipitations augmentent. Ce résultat explique le faible nombre de grains de pollen obtenu au cours de chaque mois de juillet, mois très pluvieux avec $439 \mathrm{~mm}$ d'eau en 2015 et $431,8 \mathrm{~mm}$ d'eau en 2016 qui enregistre pourtant une abondance d'espèces en pleine floraison. Ces résultats corroborent ceux obtenus par Koterbi (1997), Puc (2004), Chafai et Boughediri (2006) et Tossou et al. (2016). En effet, une forte humidité de l'atmosphère après la pluie, inhibe l'ouverture des anthères et peut modifier le poids et la vitesse de sédimentation des pollens (Chafai et Boughediri, 2006). Le pollen devient alors lourd et ne pourra plus rester suspendu un long moment dans l'air. La corrélation positive entre la température moyenne, la température maximale, la vitesse du vent et les concentrations polliniques montre d'une part qu'à température élevée, l'atmosphère est légère et favorise le maintien des grains de pollens dans l'air et d'autre part indique que le vent joue un rôle important dans le processus du maintien des grains de pollen dans l'atmosphère. 


\section{Conclusion}

Cette étude effectuée sur le contenu pollinique de l'atmosphère de la commune d'Abomey-Calavi livre des données sur deux ans. Elle donne une idée sur la caractérisation du contenu aéropollinique de cette commune. Les mois ayant connu une abondance d'espèces en pleine floraison ont enregistré un nombre élevé de grains de pollen. L’évolution journalière des pollens des espèces dominantes et leurs périodes principales de pollinisation ont été déterminées pendant chaque saison. Les corrélations entre les paramètres météorologiques et les grains de pollen indiquent que ces derniers exercent une influence sur les concentrations polliniques.

Les résultats obtenus présentent un grand intérêt pour des applications pratiques, notamment l'élaboration des calendriers polliniques et les prévisions des pics polliniques très utiles en allergologie.

Remerciements : Les auteurs remercient la Fondation Internationale des Sciences

pour la subvention de recherche $\mathrm{N}^{\circ} \mathrm{D} / 5910-1$ accordée à Frontenel Lopez TCHABI

\section{References:}

1. Andersen T.B., 1991. A model to predict the beginning of the pollen season. Grana, vol. 30, p. 269-275.

2. Achmakh L., Bouziane H., Aboulaich N., Mar Trigo M., Janati A., Kadiri M., 2015. Airbone pollen of Olea europaea L. in Tetouan (NW of Morocco). heat requierements and forescasts. Aerobiologia, vol. 31, n. 2, p. 191-199. Akoègninou A., 2004. Recherches Botaniques et écologiques sur les forêts actuelles du Bénin. Thèse d'Etat, Université de Cocody-Abidjan (Côte d'Ivoire), 326p.

3. Akoègninou A., van der Burg W.J. \& van der Maesen L. J. G., 2006. Flore analytique du Benin. Backhuys Publishers, Wageningen, 1034p.

4. Aupetit H., 1991. Les visiteurs du ciel-Guide de l'air pour l'homme volant. Ed. Rétine, Ivry, 365pp.

5. Boullard B., 1999. Dictionnaire Botanique. Edition Ellipses, 398P.

6. Bouziane H., Janati A., Kazzaz M., Ater M., 2016. Aéropalynologie de l'olivier à Tétouan (Maroc). In : Ater M. (ed.), Essalouh L. (ed), Moukhli A. (ed.). L'oléiculture au Maroc de la préhistoire à nos jours: pratiques, diversité, adaptation, usages, commerce et politiques. Montpellier: CIHEAM, 2016.p. 191-202 (Options Méditerranéennes: Série A. Séminaires Méditerranéens; n. 118) 
7. Chafai K. L., Boughediri L., 2006. Le contenu pollinique de l'atmosphère de la ville d'El Hadjar (Annaba, Algérie). Sciences et Technology C- № 24, 27-31.

8. Clot B., 2003. Plantes, Pollen \& Allergie. Les cahiers du jardin botanique. Vol 3, 34p.

9. Crossject Newletter, 2017. L'ALLERGIE, 4ème maladie chronique mondiale selon l'OMS. www.crossject.com. 1p.

10. Essien.B.C., 2014. Aeropalynological Appraisal of the Vegetation of Anyigba, Dekina Local Government Area of Kogi State, Nigeria. Unpublished. 165pp.

11. Fabiana L., Caudio F. P., 1997. One year of airbone pollen sampling in mar del plata (Argentina). Grana 36: 49-53. ISSN 0017-3134.

12. Felbert F., Clot B., 2003. Plantes, pollen \& Allergie. Les cahiers $d u$ jardin botanique. Vol 3,34p

13. Galán C, Cariñanos P, Alcazár P., Dominguez-Vilches E., 2007. Spanish Aerobiology Network (REA). Management and quality manual. Córdoba: Servicio de publicaciones de la unisersidad de Cordoba

14. García J. J., Trigo M. M., Cabezudo B., Redo M., Vega J. M., Barber D., Carmotia M. J., Cervera J. A., Toro F. J. and Miranda A., 1997. Pollinosis due to Australian pine (Casuarina): an aerobiologic and clinical study in southern Spain, Allergy, Vol. 52, pp. 11-17.

15. Green B. J., Dettmann M.-E., Yli-Panula E., Rutherford S. and Simpson R., 2004. Aeropalynology of Australian native arboreal species in Brisbane, Australia. Aerobiologia, Vol. 20, pp. 43-52.

16. Hawke P. R., Meadows M. E., 1989. Winter airspora spectra and meteorological conditions in Cape Town, South Africa. Grana 28: 187-192

17. Huynen M, Menne B, Behrendt H, Bertollini R, Bonin S, Brandao R, Brown-Fahrlander C, Clot B, D'Ambrosio C, De Nuntiis P, Ebi KL, Emberlin J, Erdei Orbanne E, Galan C, Jäger S, Mandrioli P, Menzel A, Nyenzi B, Rantio- Lehtimäki A, Ring J, Rybnicek O, TraidlHoffmann C, Van Vliet A, Voigt T, Weiland S, Wickman M., 2003. Phenology and Human Health: allergic disorders, Report of a WHO meeting, Rome, Italy, 16-17 January

18. INSAE (Institut National de la Statistique et de l'Analyse Economique)., 2013. Recensement general de la population et de l'habitat, résultats provisoires du RGPH4, MPPD, Bénin, 7p.

19. Jäger S., Nilsson S., Berggren B., Pessi A.M., Helander M. and Ramfjord H., 1996. Trends of some airbone tree pollen in the Nordic countries and Austria, 1980-1993. A comparison between Stockholm, Trondheim, Turkuand, Vienna, Grana, 35, 171-178. 
20. Jorgersen S., 1967. A method of absolue pollen counting. New pytol. 66: 489-493.

21. Kasprzyk. I., 2003. Flowering phenology and airbone pollen grains of chosen tree taxa in Rzesów. Aerobiologia, 19: 133-120.

22. Kortebi H., Hammiche V., Lamrani Z., Abed L., Larbaoui D., 1977. L'atmosphère pollinique d'Alger. Bull. Soc. Hist. Nat. Afr. Nord. Vol. 68, (3) 75-79.

23. Layachi I., 2012. Pluies polliniques et les manifestations allergiques dans le Parc National d'EL-KALA. Mémoire de DEA

24. Manju S., Swati C., 2008. Seasonal Variation of Airbone Pollen in Allahabad, India. Ann Agric Environ Med. 15: 287-293.

25. Nitiu D., 2006. Aeropalynologicanalys of la platacity (Argentina) during a 3-years period.Aerobiologia 22, 79-87

26. Puc M. and Puc M.I., 2004. Allergenic airbone grass pollen in Szczecin, Poland. Ann. Agric. Environ. Med., 11, 237-244.

27. Sowunmi M.A., 1995. Pollen grain of Nigeria plants II. Woody species. Grana 34, 120-141

28. Thibaudon M. et Lachasse C., 2005. Phénologie: intérêt et méthodes en aérobiologie. Revue française d'allergologie et d'immunologie clinique. 297-302

29. Tossou G. M., Chabi F. L., Akoègninou A., Ballouche A. et Akpagana K., 2016. Analyse pollinique de l'Atmosphère du campus d'Abomey-Calavi. Revue française d'allergologie. 56, 65-75

30. Trigo M. M., Recio M., Toro F. J., Cano M., Dopazo M. and García H., 1999. Annual variations of airborne Casuarina pollen in the Iberian Peninsula. Polen, Vol. 10, pp. 71-77..

31. Ybert J-P., 1979. Atlas des pollens de Côte d'Ivoire. ORSTOM Paris, 40 
Annexe : Tableau 1 : Spectre pollinique d'avril 2015 à avril 2017

\begin{tabular}{|c|c|c|c|c|c|c|c|c|c|c|c|c|c|c|c|}
\hline Taxa & Mois & Avril & Mai & Juin & Juillet & Août & Septembre & Octobre & Novembre & Décembre & Janvier & Février & Mars & Total & $\begin{array}{l}\text { Pourcentage } \\
(\%)\end{array}$ \\
\hline $\begin{array}{l}\text { Acacia } \\
\text { auriculiformis } \\
\text { (Leguminosae.- } \\
\text { Mimosoidae.) }\end{array}$ & $2015-2016$ & 1412 & 1229 & 1614 & 1749 & 1198 & 1314 & 937 & 1238 & 2392 & 2778 & 1412 & 1134 & 18407 & 27,84 \\
\hline \multirow{2}{*}{$\begin{array}{l}\text { Albizia lebbeck } \\
\text { (Leguminosae.- } \\
\text { Mimosoidae.) }\end{array}$} & $2015-2016$ & 158 & 36 & 17 & 47 & 21 & 78 & 33 & 32 & 32 & 11 & 5 & 21 & 491 & 0,74 \\
\hline & $2016-2017$ & 227 & 27 & 23 & 56 & 13 & 87 & 36 & 83 & 11 & 11 & 11 & 24 & 609 & 0,91 \\
\hline \multirow{2}{*}{$\begin{array}{l}\text { Alchornea } \\
\text { cordifolia } \\
\text { (Euphorbiaceae) }\end{array}$} & $2015-2016$ & 241 & 194 & 230 & 103 & 108 & 102 & 23 & 38 & 31 & 27 & 11 & 35 & 1143 & 1,73 \\
\hline & $2016-2017$ & 364 & 76 & 122 & 89 & 86 & 67 & 52 & 27 & 32 & 31 & 42 & 43 & 1031 & 1,54 \\
\hline \multirow[t]{2}{*}{ Apocynaceae-type } & $2015-2016$ & 111 & 144 & 119 & 68 & 58 & 43 & 21 & 21 & 43 & 31 & 75 & 34 & 768 & 1,16 \\
\hline & $2016-2017$ & 234 & 112 & 21 & 43 & 62 & 36 & 33 & 67 & 51 & 24 & 23 & 42 & 748 & 1,11 \\
\hline \multirow{2}{*}{$\begin{array}{l}\text { Bougainvillea } s p \\
\text { (Nyctaginaceae) }\end{array}$} & $2015-2016$ & 169 & 42 & 115 & 21 & 18 & 32 & 57 & 37 & 47 & 67 & 47 & 64 & 716 & 1,08 \\
\hline & 2016-2017 & 281 & 9 & 8 & 19 & 26 & 42 & 71 & 42 & 46 & 56 & 41 & 76 & 717 & 1,09 \\
\hline $\begin{array}{c}\text { Carica papaya } \\
\text { (Caricaceae) }\end{array}$ & $2015-2016$ & 0 & 0 & 0 & 0 & 0 & 0 & 0 & 0 & 0 & 0 & 0 & 0 & 0 & 0 \\
\hline \multirow{2}{*}{$\begin{array}{l}\text { Casuarina } \\
\text { equisetifolia } \\
\text { (Casuarinaceae) }\end{array}$} & $2015-2016$ & 0 & 0 & 0 & 0 & 0 & 31 & 21 & 12 & 50 & 21 & 33 & 64 & 232 & 0,35 \\
\hline & 2016-2017 & 0 & 0 & 0 & 0 & 0 & 0 & 11 & 0 & 49 & 16 & 43 & 67 & 186 & 0,29 \\
\hline \multirow{2}{*}{$\begin{array}{l}\text { Caesalpinia } \\
\text { pulcherima } \\
\text { (Leguminosae- } \\
\text { Caesalpinoideae.) }\end{array}$} & $2015-2016$ & 90 & 274 & 126 & 86 & 21 & 97 & 78 & 82 & 54 & 62 & 67 & 43 & 1053 & 1,59 \\
\hline & 2016-2017 & 180 & 94 & 108 & 88 & 19 & 91 & 75 & 99 & 71 & 78 & 49 & 64 & 1016 & 1,51 \\
\hline \multirow{2}{*}{$\begin{array}{l}\text { Ceiba pentadra } \\
\text { (Bombacaceae) }\end{array}$} & $2015-2016$ & 158 & 14 & 4 & 0 & 0 & 44 & 48 & 48 & 65 & 46 & 76 & 76 & 579 & 0,87 \\
\hline & $2016-2017$ & 349 & 3 & 0 & 0 & 0 & 61 & 49 & 31 & 80 & 56 & 82 & 74 & 785 & 1,17 \\
\hline \multirow{2}{*}{$\begin{array}{c}\text { Celosia laxa } \\
\text { (Amaranthaceae) }\end{array}$} & $2015-2016$ & 95 & 104 & 138 & 102 & 121 & 98 & 132 & 89 & 108 & 67 & 79 & 45 & 1178 & 1,78 \\
\hline & 2016-2017 & 89 & 128 & 112 & 76 & 96 & 122 & 86 & 98 & 112 & 89 & 73 & 67 & 1148 & 1,72 \\
\hline \multirow{2}{*}{$\begin{array}{c}\text { Cocos nucifera } \\
\text { (Arecaceae) }\end{array}$} & $2015-2016$ & 188 & 122 & 151 & 96 & 56 & 73 & 87 & 67 & 63 & 67 & 93 & 83 & 1146 & 1,73 \\
\hline & $2016-2017$ & 194 & 125 & 108 & 21 & 61 & 67 & 69 & 71 & 71 & 89 & 67 & 62 & 1005 & 1,5 \\
\hline Combretum & $2015-2016$ & 1264 & 1181 & 756 & 259 & 645 & 978 & 567 & 472 & 671 & 998 & 992 & 632 & 9415 & 14,24 \\
\hline
\end{tabular}




\begin{tabular}{|c|c|c|c|c|c|c|c|c|c|c|c|c|c|c|c|}
\hline $\begin{array}{l}\text { indicum } \\
\text { (Combretaceae) }\end{array}$ & 2016-2017 & 1286 & 866 & 225 & 324 & 1143 & 837 & 678 & 231 & 1315 & 1002 & 918 & 673 & 9498 & 14,20 \\
\hline \multirow{2}{*}{$\begin{array}{l}\text { Chromolaena } \\
\text { odorata } \\
\text { (Asteraceae) }\end{array}$} & $2015-2016$ & 83 & 11 & 0 & 108 & 121 & 147 & 131 & 32 & 67 & 91 & 86 & 64 & 941 & 1,42 \\
\hline & $2016-2017$ & 295 & 9 & 134 & 11 & 16 & 43 & 63 & 41 & 89 & 87 & 72 & 36 & 896 & 1,33 \\
\hline \multirow{2}{*}{$\begin{array}{l}\text { Crotalaria retusa } \\
\text { (Leguminosae- } \\
\text { Papilionoideae) }\end{array}$} & $2015-2016$ & 90 & 83 & 9 & 32 & 32 & 32 & 17 & 31 & 42 & 23 & 71 & 42 & 504 & 0,76 \\
\hline & 2016-2017 & 378 & 212 & 7 & 13 & 11 & 19 & 19 & 33 & 41 & 23 & 43 & 41 & 840 & 1,26 \\
\hline \multirow{2}{*}{$\begin{array}{l}\text { Cyperus rotundus } \\
\text { (Cyperaceae) }\end{array}$} & $2015-2016$ & 0 & 0 & 0 & 31 & 46 & 56 & 71 & 22 & 12 & 34 & 22 & 31 & 325 & 0,49 \\
\hline & $2016-2017$ & 40 & 0 & 0 & 21 & 34 & 62 & 21 & 13 & 16 & 42 & 21 & 39 & 309 & 0,46 \\
\hline \multirow{2}{*}{$\begin{array}{l}\text { Delonix regia } \\
\text { (Leguminosae- } \\
\text { Caesalpinoideae) }\end{array}$} & $2015-2016$ & 133 & 148 & 98 & 67 & 83 & 61 & 41 & 31 & 41 & 43 & 24 & 33 & 803 & 1,21 \\
\hline & $2016-2017$ & 191 & 101 & 32 & 72 & 61 & 39 & 38 & 24 & 37 & 45 & 31 & 35 & 706 & 1,05 \\
\hline \multirow{2}{*}{$\begin{array}{l}\text { Dichapetalum } \\
\text { madagascariensis } \\
\text { (Dichapetalaceae) }\end{array}$} & $2015-2016$ & 4 & 184 & 277 & 212 & 45 & 47 & 47 & 73 & 68 & 43 & 45 & 17 & 1062 & 1,60 \\
\hline & 2016-2017 & 94 & 103 & 188 & 198 & 48 & 52 & 65 & 82 & 40 & 36 & 33 & 21 & 960 & 1,43 \\
\hline \multirow{2}{*}{$\begin{array}{l}\text { Dichrostachys } \\
\text { cinerea } \\
\text { (Leguminosae- } \\
\text { mimosoidae) }\end{array}$} & $2015-2016$ & 8 & 4 & 41 & 43 & 47 & 82 & 79 & 57 & 47 & 39 & 41 & 41 & 529 & 0,80 \\
\hline & $2016-2017$ & 18 & 3 & 23 & 51 & 82 & 49 & 29 & 66 & 37 & 31 & 28 & 42 & 459 & 0,69 \\
\hline \multirow{2}{*}{$\begin{array}{c}\text { Diospyros type } \\
\text { (Ebenaceae) }\end{array}$} & $2015-2016$ & 158 & 24 & 32 & 21 & 23 & 42 & 47 & 38 & 34 & 51 & 56 & 43 & 569 & 0,86 \\
\hline & $2016-2017$ & 144 & 9 & 33 & 19 & 31 & 51 & 46 & 37 & 42 & 61 & 51 & 36 & 587 & 0,88 \\
\hline \multirow{2}{*}{$\begin{array}{l}\text { Elaeis guineensis } \\
\text { (Arecaceae) }\end{array}$} & $2015-2016$ & 212 & 320 & 230 & 346 & 341 & 311 & 341 & 416 & 312 & 415 & 289 & 508 & 4041 & 6,11 \\
\hline & $2016-2017$ & 335 & 248 & 328 & 431 & 287 & 332 & 321 & 403 & 314 & 441 & 301 & 451 & 4192 & 6,26 \\
\hline \multirow{2}{*}{$\begin{array}{l}\text { Grewia } \\
\text { carpinifolia } \\
\text { (Tiliaceae) }\end{array}$} & $2015-2016$ & 0 & 36 & 40 & 32 & 41 & 31 & 45 & 31 & 32 & 43 & 32 & 43 & 406 & 0,61 \\
\hline & 2016-2017 & 30 & 28 & 38 & 27 & 29 & 18 & 18 & 21 & 39 & 32 & 25 & 43 & 348 & 0,52 \\
\hline \multirow{2}{*}{$\begin{array}{l}\text { Hoslundia } \\
\text { opposita } \\
\text { (Lamiaceae) }\end{array}$} & $2015-2016$ & 31 & 40 & 47 & 38 & 46 & 48 & 67 & 71 & 45 & 56 & 21 & 41 & 551 & 0,83 \\
\hline & $2016-2017$ & 40 & 37 & 32 & 46 & 48 & 46 & 61 & 43 & 46 & 53 & 31 & 36 & 565 & 0,84 \\
\hline \multirow{2}{*}{$\begin{array}{l}\text { Ixora } \\
\text { brachyopoda } \\
\text { (Rubiaceae) }\end{array}$} & $2015-2016$ & 43 & 31 & 18 & 31 & 21 & 31 & 34 & 34 & 45 & 47 & 31 & 23 & 389 & 0,59 \\
\hline & 2016-2017 & 30 & 21 & 26 & 42 & 27 & 26 & 36 & 42 & 48 & 56 & 43 & 28 & 425 & 0,63 \\
\hline \multirow{2}{*}{$\begin{array}{l}\text { Jatropha curcas } \\
\text { (Euphorbiaceae) }\end{array}$} & $2015-2016$ & 4 & 11 & 4 & 1 & 1 & 2 & 6 & 6 & 3 & 6 & 2 & 3 & 49 & 0,07 \\
\hline & $2016-2017$ & 18 & 16 & 0 & 0 & 1 & 3 & 7 & 12 & 6 & 5 & 3 & 8 & 79 & 0,12 \\
\hline \multirow{2}{*}{$\begin{array}{c}\text { Lantana camara } \\
\text { (Verbenaceae) }\end{array}$} & $2015-2016$ & 11 & 11 & 94 & 17 & 8 & 13 & 11 & 6 & 6 & 6 & 7 & 8 & 198 & 0,3 \\
\hline & $2016-2017$ & 11 & 87 & 11 & 11 & 4 & 14 & 5 & 6 & 7 & 3 & 2 & 4 & 165 & 0,25 \\
\hline Lippia & $2015-2016$ & 0 & 0 & 0 & 0 & 0 & 0 & 0 & 0 & 0 & 0 & 0 & 4 & 4 & 0 \\
\hline
\end{tabular}




\begin{tabular}{|c|c|c|c|c|c|c|c|c|c|c|c|c|c|c|c|}
\hline $\begin{array}{l}\text { multifolora(Verbe } \\
\text { naceae) }\end{array}$ & $2016-2017$ & 2 & 1 & 0 & 0 & 0 & 0 & 0 & 0 & 0 & 10 & 21 & 3 & 37 & 0,05 \\
\hline \multirow{2}{*}{$\begin{array}{l}\text { Mallotus } \\
\text { oppositifolius } \\
\text { (Euphorbiaceae) }\end{array}$} & $2015-2016$ & 0 & 223 & 104 & 43 & 36 & 43 & 42 & 21 & 31 & 45 & 31 & 56 & 675 & 1,02 \\
\hline & $2016-2017$ & 22 & 201 & 72 & 28 & 51 & 44 & 41 & 41 & 43 & 38 & 57 & 32 & 670 & 1 \\
\hline \multirow[t]{2}{*}{ Malvaceae } & $2015-2016$ & 0 & 0 & 0 & 0 & 11 & 5 & 34 & 0 & 0 & 9 & 8 & 1 & 68 & 0,1 \\
\hline & $2016-2017$ & 3 & 0 & 0 & 12 & 17 & 11 & 31 & 0 & 0 & 0 & 8 & 7 & 89 & 0,13 \\
\hline \multirow{2}{*}{$\begin{array}{l}\text { Mangifera indica } \\
\text { (Anacardiaceae) }\end{array}$} & $2015-2016$ & 126 & 169 & 104 & 121 & 46 & 46 & 39 & 51 & 47 & 37 & 42 & 31 & 859 & 1,3 \\
\hline & $2016-2017$ & 148 & 122 & 101 & 123 & 43 & 42 & 46 & 62 & 36 & 34 & 37 & 32 & 826 & 1,23 \\
\hline \multirow{2}{*}{$\begin{array}{l}\text { Mariscus } \\
\text { alternifolius } \\
\text { (Cyperaceae) }\end{array}$} & $2015-2016$ & 22 & 14 & 0 & 0 & 0 & 3 & 10 & 0 & 7 & 6 & 4 & 5 & 71 & 0,1 \\
\hline & 2016-2017 & 43 & 41 & 0 & 0 & 0 & 4 & 0 & 3 & 12 & 7 & 6 & 6 & 122 & 0,18 \\
\hline \multirow{2}{*}{$\begin{array}{l}\text { Morinda lucida } \\
\text { (Rubiaceae) }\end{array}$} & $2015-2016$ & 0 & 18 & 4 & 3 & 5 & 22 & 13 & 21 & 1 & 16 & 3 & 21 & 127 & 0,19 \\
\hline & $2016-2017$ & 30 & 39 & 4 & 4 & 13 & 31 & 14 & 32 & 0 & 19 & 6 & 41 & 233 & 0,35 \\
\hline \multirow{2}{*}{$\begin{array}{l}\text { Paullinia pinnata } \\
\text { (Sapindaceae) }\end{array}$} & $2015-2016$ & 0 & 140 & 86 & 42 & 16 & 26 & 31 & 43 & 46 & 31 & 41 & 45 & 547 & 0,82 \\
\hline & 2016-2017 & 22 & 141 & 71 & 61 & 33 & 13 & 31 & 12 & 41 & 21 & 46 & 61 & 553 & 0,82 \\
\hline \multirow{2}{*}{$\begin{array}{l}\text { Phaulopsis ciliata } \\
\text { (Acanthaceae) }\end{array}$} & $2015-2016$ & 101 & 140 & 155 & 64 & 48 & 26 & 31 & 47 & 59 & 46 & 38 & 51 & 806 & 1,22 \\
\hline & 2016-2017 & 0 & 132 & 87 & 47 & 47 & 36 & 44 & 61 & 61 & 42 & 37 & 55 & 649 & 0,97 \\
\hline \multirow{2}{*}{$\begin{array}{l}\text { Phoenix reclinata } \\
\text { (Arecaceae) }\end{array}$} & $2015-2016$ & 108 & 104 & 122 & 122 & 11 & 46 & 45 & 61 & 43 & 33 & 31 & 23 & 538 & 0,81 \\
\hline & $2016-2017$ & 133 & 89 & 78 & 11 & 15 & 67 & 34 & 34 & 44 & 37 & 33 & 21 & 627 & 0,94 \\
\hline \multirow{2}{*}{$\begin{array}{l}\text { Pinus sp } \\
\text { (Pinaceae) }\end{array}$} & $2015-2016$ & 0 & 0 & 0 & 1 & 31 & 17 & 0 & 0 & 0 & 11 & 23 & 30 & 113 & 0,17 \\
\hline & $2016-2017$ & 0 & 0 & 0 & 12 & 21 & 16 & 0 & 0 & 2 & 13 & 11 & 23 & 98 & 0,15 \\
\hline \multirow[t]{2}{*}{ Poaceae-type } & $2015-2016$ & 1048 & 1863 & 1289 & 1208 & 917 & 1121 & 1021 & 918 & 792 & 934 & 998 & 1247 & 13356 & 20,20 \\
\hline & $2016-2017$ & 1168 & 1718 & 2139 & 931 & 1032 & 1078 & 1032 & 809 & 1031 & 1011 & 1135 & 1098 & 14182 & 21,20 \\
\hline \multirow{2}{*}{$\begin{array}{l}\text { Ricinus communis } \\
\text { (Euphorbiaceae) }\end{array}$} & $2015-2016$ & 72 & 68 & 72 & 47 & 46 & 76 & 29 & 45 & 32 & 42 & 46 & 61 & 636 & 0,96 \\
\hline & $2016-2017$ & 158 & 61 & 68 & 38 & 42 & 54 & 43 & 44 & 16 & 32 & 62 & 54 & 672 & 1 \\
\hline \multirow{2}{*}{$\begin{array}{l}\text { Reissantia indica } \\
\text { (Celastraceae) }\end{array}$} & $2015-2016$ & 29 & 151 & 97 & 51 & 47 & 59 & 21 & 32 & 31 & 45 & 58 & 43 & 664 & 1 \\
\hline & $2016-2017$ & 52 & 82 & 87 & 52 & 61 & 56 & 23 & 34 & 42 & 47 & 48 & 46 & 700 & 1,04 \\
\hline \multirow{2}{*}{$\begin{array}{l}\text { Sapotaceae/Melia } \\
\text { ceae-type }\end{array}$} & $2015-2016$ & 119 & 184 & 27 & 41 & 62 & 43 & 26 & 23 & 29 & 8 & 43 & 32 & 547 & 0,83 \\
\hline & 2016-2017 & 155 & 88 & 32 & 48 & 21 & 23 & 14 & 12 & 18 & 11 & 41 & 28 & 550 & 0,82 \\
\hline \multirow{2}{*}{$\begin{array}{l}\text { Senna siamea } \\
\text { (Leguminosae- } \\
\text { Caesalpinoideae) }\end{array}$} & $2015-2016$ & 43 & 187 & 180 & 37 & 31 & 24 & 14 & 14 & 21 & 23 & 22 & 27 & 623 & 0,94 \\
\hline & $2016-2017$ & 68 & 103 & 32 & 52 & 36 & 21 & 16 & 12 & 29 & 21 & 26 & 21 & 437 & 0,65 \\
\hline \multirow{2}{*}{$\begin{array}{l}\text { Talinum } \\
\text { triangulare } \\
\text { (Portulacaceae) }\end{array}$} & $2015-2016$ & 8 & 1 & 1 & 4 & 1 & 5 & 2 & 7 & 1 & 6 & 4 & 4 & 44 & 0,06 \\
\hline & 2016-2017 & 2 & 6 & 1 & 1 & 1 & 6 & 0 & 6 & 9 & 8 & 4 & 7 & 51 & 0,08 \\
\hline Tephrosia & $2015-2016$ & 112 & 41 & 7 & 32 & 32 & 21 & 7 & 35 & 11 & 14 & 6 & 11 & 329 & 0,5 \\
\hline
\end{tabular}




\begin{tabular}{|c|c|c|c|c|c|c|c|c|c|c|c|c|c|c|c|}
\hline $\begin{array}{l}\text { purpurea } \\
\text { (Leguminoseae- } \\
\text { papilionoïdeae) }\end{array}$ & $2016-2017$ & 130 & 82 & 7 & 75 & 8 & 18 & 9 & 33 & 7 & 12 & 8 & 9 & 398 & 0,59 \\
\hline \multirow{2}{*}{$\begin{array}{l}\text { Terminalia } \\
\text { mantaty } \\
\text { (Combretaceae) }\end{array}$} & 2015-2016 & 0 & 0 & 0 & 0 & 32 & 21 & 38 & 10 & 6 & 0 & 0 & 0 & 107 & 0,16 \\
\hline & 2016-2017 & 0 & 1 & 0 & 0 & 26 & 13 & 22 & 13 & 4 & 0 & 0 & 3 & 82 & 0,12 \\
\hline \multirow{2}{*}{$\begin{array}{l}\text { Thumbergia } \\
\text { erecta } \\
\text { (Acanthaceae) }\end{array}$} & $2015-2016$ & 0 & 14 & 11 & 6 & 6 & 5 & 8 & 4 & 14 & 8 & 10 & 32 & 118 & 0,18 \\
\hline & $2016-2017$ & 7 & 41 & 8 & 7 & 56 & 7 & 9 & 25 & 16 & 4 & 7 & 21 & 208 & 0,31 \\
\hline \multirow{2}{*}{$\begin{array}{l}\text { Tridax } \\
\text { procumbens } \\
\text { (Asteraceae) }\end{array}$} & $2015-2016$ & 101 & 97 & 83 & 21 & 8 & 12 & 10 & 9 & 14 & 7 & 11 & 9 & 382 & 0,57 \\
\hline & $2016-2017$ & 104 & 210 & 27 & 16 & 8 & 9 & 9 & 12 & 8 & 5 & 14 & 6 & 428 & 0,64 \\
\hline
\end{tabular}

